\title{
MD simulation of dislocation mobility during cutting with diamond tip on silicon
}

\author{
Q.H. Tang* \\ State Key Laboratory of Nonlinear Mechanics, Institute of Mechanics, Chinese Academy of Sciences, Beijing 100080, PR China
}

\section{A R T I C L E I N F O}

\section{Keywords:}

Molecular dynamics simulation

Atomic force microscopy

Silicon

Dislocation

Pin tool

\begin{abstract}
A B S T R A C T
By means of Tersoff and Morse potentials, a three-dimensional molecular dynamics simulation is performed to study atomic force microscopy cutting on silicon monocrystal surface. The interatomic forces between the workpiece and the pin tool and the atoms of workpiece themselves are simulated. Two partial edge dislocations are introduced into workpiece $\mathrm{Si}$, it is found that the motion of dislocations does not occur during the atomic force microscopy cutting processing. Simulation results show that the shear stress acting on dislocations is far below the yield strength of $\mathrm{Si}$.
\end{abstract}

(c) 2008 Elsevier Ltd. All rights reserved.

\section{Introduction}

Silicon crystals are the most popular material used in microelectronic devices, where mechanical behaviors, such as crack extension, dislocations motion, and transition from brittle to ductile state, are important to the performance of devices. Significant works have been done to understand these mechanical behavior of silicon by different methods [1-8].

In general crystalline $\mathrm{Si}$ is brittle and shows no plasticity below the ductile-brittle transition temperature (DBTT) when the crystal is deformed in bulk form [1]. However, two exceptions on this have been reported. One is that at room temperature less than DBTT, Rabier et al. [2] performed experiments of silicon by transmission electron microscopy (TEM) at high confining pressures, the dislocations movement has been observed at $15 \mathrm{GPa}$ in the metallic phase. And the other is the plastic deformation identified under indentation [3,4]. Asaoka et al. [4] observed shuffle dislocation activated under indentation at $77 \mathrm{~K}$ by the technique of TEM and in situ heating. At present theoretical calculations have been carried out to predict the mechanical properties of silicon on the

\footnotetext{
* Corresponding author. Tel.: +86 01062613546; fax: +86 0106259511.

E-mail address: qhtang@lnm.imech.ac.cn
}

nanometer scale. Kaxiras and Duesbery [5] calculated the unstable stacking fault energy by using the density functional theory (DFT), which shows that the dislocation structure in $\mathrm{Si}$ is available. The $\mathrm{AB}$ initio calculation performed by Perez and Gumbsch [6] is used to study the cleavage anisotropy in silicon, the different lattice trapping for different crack propagation directions can explain the cleavage observed in experiment. By employing multiscale molecular dynamics (MD) simulation with a tight-binding description of bonding near the crack tip embedded in an empirical-potential region, Bernstein and Hess [7] pointed out that the lattice trapping plays an important role in the brittle-to-ductile transition. One can understand that such quantum-mechanical calculations are too expensive in computational cost and cannot be applied to the system with several thousands of particles. The classical MD technique with empirical-potential should have a role on investigation of mechanical properties of silicon. Tanaka et al. [8] studied fracture toughness of silicon crystals by indentation method with experiment and MD simulation. The surface energy and fracture toughness $K$ were calculated. Tang and Zhang [9] discussed the mechanical effect of water on the deformation of silicon monocrystals under nano-indentation, and pointed out that the presence of water reduces indentor-silicon adhesion.

The atomic force microscope (AFM) has been developed as a tool which allows routine investigation of 
surface structures [10]. It is used in the surface processing of nanoscale semiconductor devices [11-13]. Tremendous efforts have been thrown into controlling the surface modification of materials. The merit of the AFM-based lithographic techniques is that the fabrication resolution can be extended to the nanometer scale. But the lithographic mechanism on the atomic scale is not well understood. Employing MD simulation is both proper and beneficial for us to trace the behavior of atoms and understand deformation mechanism of the atomic scale.

The nucleation and motion of dislocations in Si are hot topic because the cores harbor localized in-gap electronic levels which can destroy the electrical properties of a device. If dislocations are not mobile, device logic can be made to avoid the dislocated areas. And an optoelectronic device based on strained lattice adds additional motivation to the understanding of dislocation nucleation and mobility [14].

The main objective of this article is to study the stability of dislocation structure in workpiece silicon deformation during the cutting process. The 3D MD is performed under the canonical ensemble (NVT) with the environment temperature $30 \mathrm{~K}$.

\section{Computation and modeling}

\subsection{Initial model}

In the present simulation, a workpiece and a pin tool are assumed to consist, respectively, of monocrystalline silicon and rigid diamond, as shown in Fig. 1. The dimension of the silicon workpiece is constructed sufficiently large to eliminate boundary effects. According to the previous simulation [15], the size of workpiece of $12.8 \times 7.3 \times 13.2 \mathrm{~nm}^{3}$ along $x, y$, and $z$ directions is enough. The coordinate system is selected to be $x, y$, and $z$ axes along [ $\left.\begin{array}{lll}1 & \overline{1} & 0\end{array}\right],\left[\begin{array}{lll}1 & 1 & 1\end{array}\right]$, and [ $\left.\begin{array}{lll}1 & 1 & \overline{2}\end{array}\right]$, respectively.

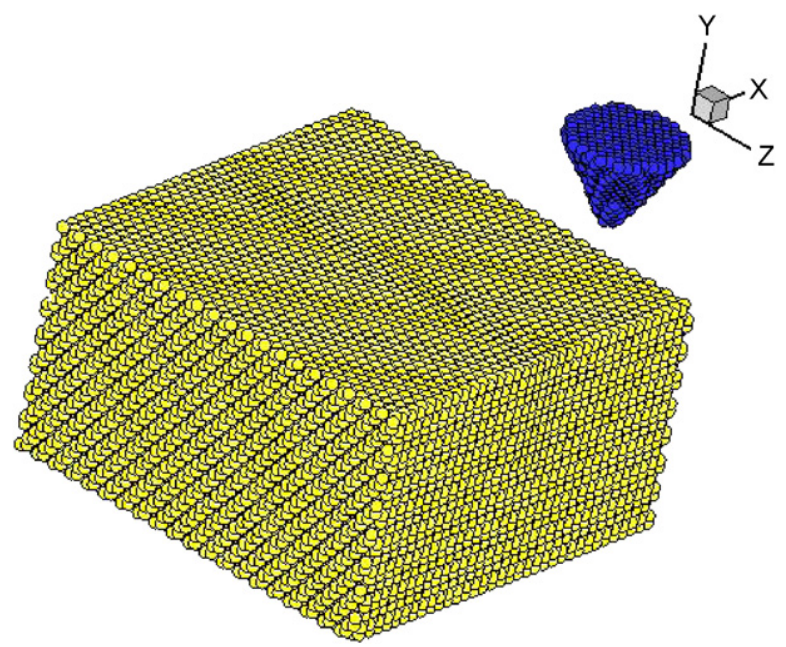

Fig. 1. Molecular dynamics simulation model of silicon monocrystal and diamond pin tool. The size of workpiece silicon is $12.8 \times 7.3 \times 13.2 \mathrm{~nm}^{3}$.

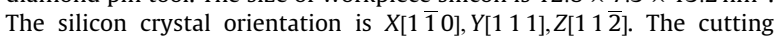
velocity of pin tool is $110 \mathrm{~m} / \mathrm{s}$.
Two partial dislocations on the slip place (111), with the burgers vector along [1 $\overline{1} 0]$, are introduced into workpiece Si. The diamond tool is $3 \mathrm{~nm}$ high and has a 60 tool angle with a tip radius of $0.34 \mathrm{~nm}$. The workpiece is made up of 68185 silicon atoms and tool is made of 1945 carbon atoms. The periodic boundary condition in $[16,17]$ is used in the transverse $z$ direction and one layer of atoms at the left and three layers of atoms at the bottom sides of silicon are fixed in space [18]. The pin tool is moved on the silicon surface (111) along the negative $x$ direction.

\subsection{Interatomic potentials}

There are several categories of existing potential models for silicon, e.g. the Tersoff type [19,20] and the Stillinger-Weber (S-W) two- and three-body potentials [21]. Tersoff potential has been widely used for MD simulation.

For covalent silicon, bond strength and the directionality of bond are important. Tersoff potential can well describe the properties of silicon and give reliable results of simulation. Hence, Tersoff potential is selected in our work to indicate the interaction between silicon atoms $[19,20]$.

The interaction between silicon atoms and diamond pin tool atoms is modeled by the Morse potential [17]

$\phi\left(r_{i j}\right)=\lambda_{1} D\left[\exp \left(-2 \lambda_{2} \alpha\left(r_{i j}-r_{0}\right)\right)-2 \exp \left(-\lambda_{2} \alpha\left(r_{i j}-r_{0}\right)\right)\right]$

where parameters such as $D, \alpha$, and $r_{0}$ are shown in Table 1.

\subsection{Temperature condition and loading method}

Initial velocities of atoms are specified based on the Maxwellian distribution corresponding to a given temperature, $T_{0}=30 \mathrm{~K}$, and the magnitudes may be adjusted so as to keep constant temperature in the system according to

$v_{i}^{\text {new }}=\left\{\frac{3 N \kappa_{\mathrm{B}} T_{0}}{2}\left[\sum_{i=1}^{N} \frac{m_{i}\left(v_{i}^{\text {old }}\right)^{2}}{2}\right]^{-1}\right\}^{1 / 2} v_{i}^{\text {old }}$

where $v_{i}$ is the velocity of atom $i, T_{0}$ is a specified temperature, $\kappa_{\mathrm{B}}$ is Boltzmann's constant, and $N$ is the number of atoms of workpiece in which fixed atoms are excluded [17]. The time integration of motion is performed by the fifth Gear's predictor-corrector method [22], time step $\Delta t=1.0 \mathrm{fs}$.

Table 1

Parameters in Morse potential

\begin{tabular}{ll}
\hline Parameters & C-Si \\
\hline$D(\mathrm{eV})$ & 0.435 \\
$\alpha\left(\mathrm{nm}^{-1}\right)$ & 46.487 \\
$r_{0}$ & 0.19475 \\
$\lambda_{1}$ & 1 \\
$\lambda_{2}$ & 1 \\
\hline
\end{tabular}




\section{Results and discussion}

The simulation system consists of a silicon workpiece and a diamond tool. The experiment shows that the hardness of silicon is $9.3 \mathrm{GPa}$ [23] and diamond $78.96 \mathrm{GPa}$ [24], one can assume that diamond tip does not deform in the process of cutting the workpiece surface. After 5000 MD steps relaxation at an environment temperature of $30 \mathrm{~K}$, a stable structure of workpiece occurs; and the pin tool begins to cut along the $-X$ direction at a velocity of $110 \mathrm{~m} / \mathrm{s}$. It is noted that the cutting speed is unrealistic high by comparison with AFM experiments. However, previous results $[8,9,15]$ have confirmed a little difference on deformation characteristics such as surface quality at a cutting speeds 20 and $200 \mathrm{~m} / \mathrm{s}$, respectively. The depth of diamond tip plunging into silicon workpiece is $5 \mathrm{~nm}$, which is similar to that used by Fang and Weng [15].

\subsection{Analysis of force}

The force variations of the nano-lithographic process versus time are shown in Fig. 2. The force components of $F_{x}$ is the cutting force which is higher than those of thrust force $F_{y}$ and width direction force $F_{z}$. In the cutting process, the width direction force $F_{z}$ is with a little variation; and the cutting force and thrust force $F_{x}$ and $F_{y}$ are quite important. $F_{y}$ is along the vertical direction, and the enough magnitude of force component in vertical direction can keep cut depth of pin tool. The force $F_{X}$ along cutting direction is larger than other two force components. The ratio of $F_{x}$ to $F_{y}$ is defined as the cutting resistance $f_{\mathrm{R}}$ [10]. $f_{\mathrm{R}} \gg 1$ means that the cutting resistance is so large that it is difficult to cut silicon material with diamond tool. Similar simulation results were obtained by Kim et al. [24] for crystal copper. In their simulations, six different crystal setup were carried out and the results show the resistance force $f_{\mathrm{R}} \gg 1$.

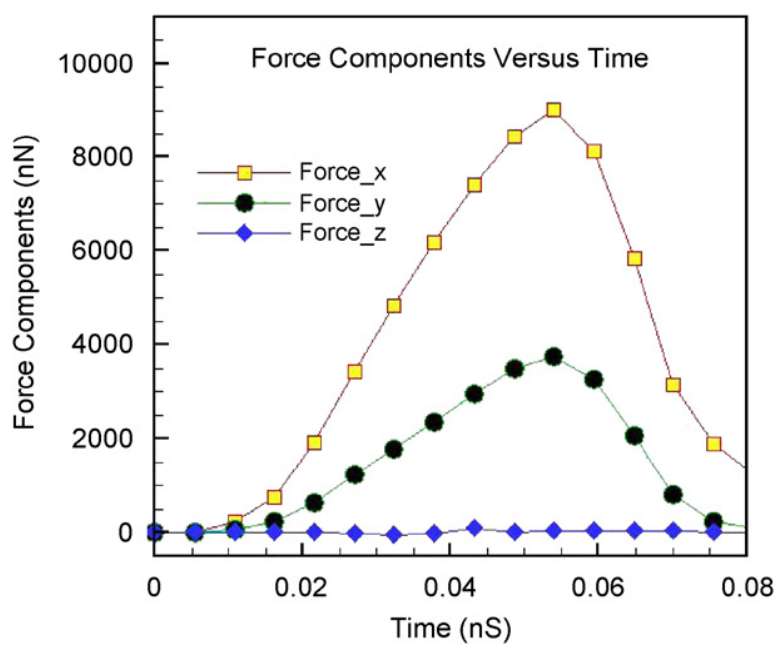

Fig. 2. Curves of force components versus running time.

\subsection{Stable dislocation structure}

Hornstra [25] proposed several possible structures of dislocations in the diamond lattice. The most probable type of partial dislocations and the mechanism of their formation were discussed, and pointed out that of the partial dislocations only two types, $a / 6[1 \overline{2} 1](111)$ and $a / 6[\overline{1} \overline{1} 2]\left(\begin{array}{lll}1 & 1 & 1\end{array}\right)$, are important. Their unstable stacking energies were calculated by Kaxiras and Duesbery [5] using the density functional theory (DFT). They showed that the nucleation and motion of partial edge dislocations on the (111) plane is favorable. Fig. 3a shows the two partial edge dislocations placed at $x=-5$ and 5 , respectively. The stable state of dislocation structure is obtained after running $5000 \mathrm{MD}$ steps at $30 \mathrm{~K}$. After pin tool cut through the dislocation region, the atomic

a

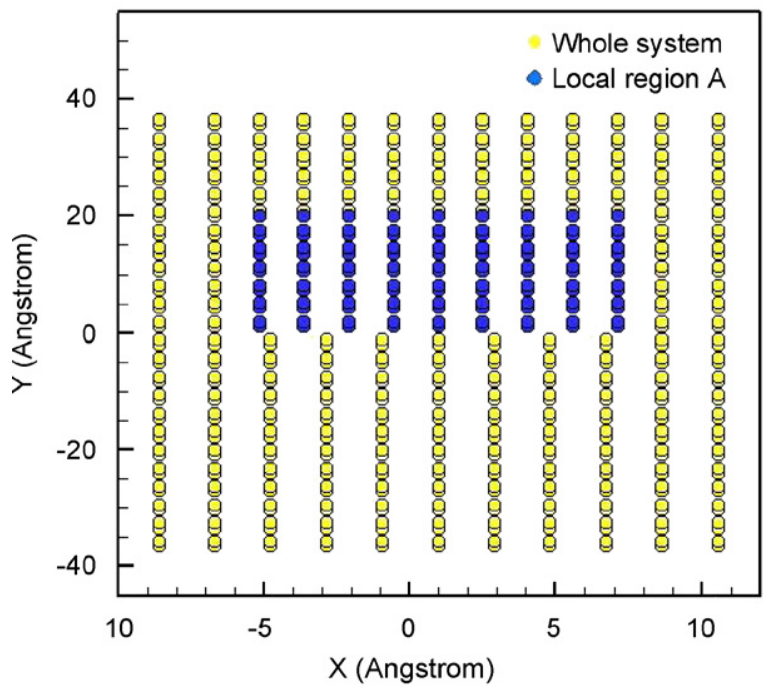

b

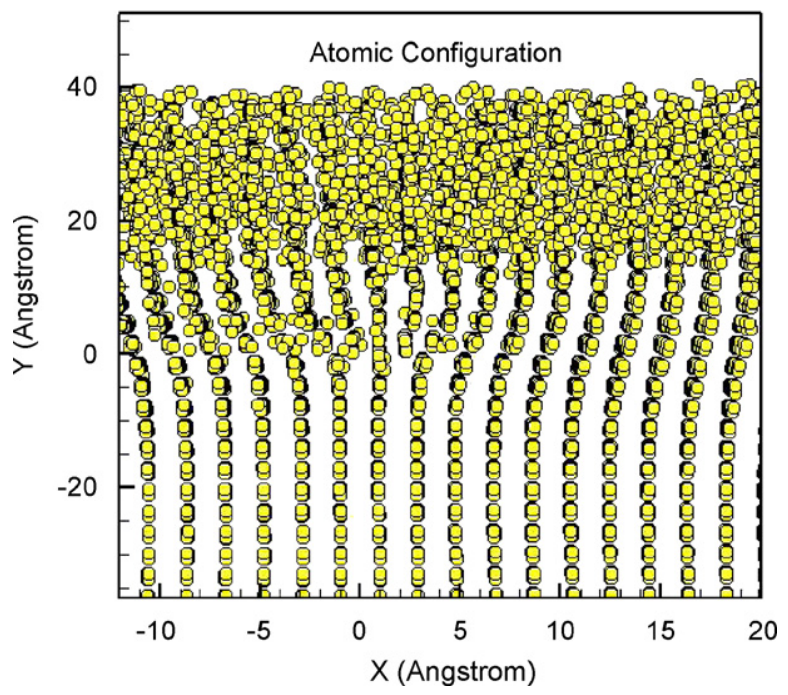

Fig. 3. Diagram of atomic structure. (a) Initial atom configuration with local region A just above the slip plane of dislocation. (b) Atom configuration after the pin tool cutting. 
configuration with the dislocation core is plotted in Fig. 3b. Compared Figs. 3a with b, it is found that the two partial edge dislocations do not move after AFM loading. One knows that, to make the crystal dislocation slip or glide on the slip plane, the shear stress acting on the dislocations should be big enough. A stress analysis is required to explain our $\mathrm{MD}$ simulation results. A local region $A$ just above the dislocation line is selected, see Fig. 3a. The average stresses of the local region $A$ are obtained during the cutting process. Fig. 4 gives the variation of average stresses versus simulation time. The normal stresses are negative, which means that the workpiece $\mathrm{Si}$ is in the state of compression. The cutting stress is the biggest, up to $-32 \mathrm{GPa}$, the thrust stress is the second, up to $-20 \mathrm{GPa}$, and the width stress is about $-10 \mathrm{GPa}$. It is noted that the shear stress is quite small, and its value is just $-0.35 \mathrm{GPa}$ when time is $0.02 \mathrm{~ns}$. Such a smallshear stress do not make the partial dislocation move. Based on the Peierls-Nabarro model Ren et al. [14] pointed out the yield strength of Si is $0.1-0.5 \mu$, where $\mu=$ 68.1 GPa is the shear modulus [26]. It is obvious to see that the theoretical result is nearly 20 times higher than that of our simulation. The similar result was given by Duesbery and Joos [27], the Peierls stresses of $0.56 \mu$ for $30^{\circ}$ glide partial and the shuffle screw dislocations were calculated by the Peierls model.

\subsection{Potential energy and temperature}

In order to understand the behaviors of workpiece silicon under cutting loading, some physical qualities such as the potential energy and temperature variation of the local area near the pin tip are analyzed. The local region $B$ of dynamic assemble of atom array near pin tool is constructed, see Fig. 5. The potential energy variation is plotted in Fig. 6a. The mean potential energy per atom in the workpiece is about $-4.45 \mathrm{eV}$, and a small increment is observed with MD steps due to pin tool loading. There is

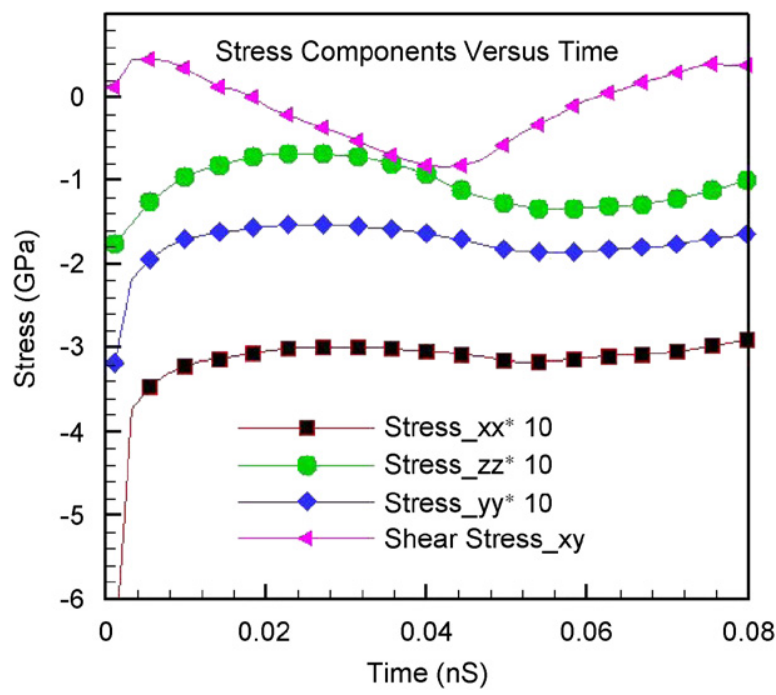

Fig. 4. Comparion of average normal stress with shear stress at local region $A$.

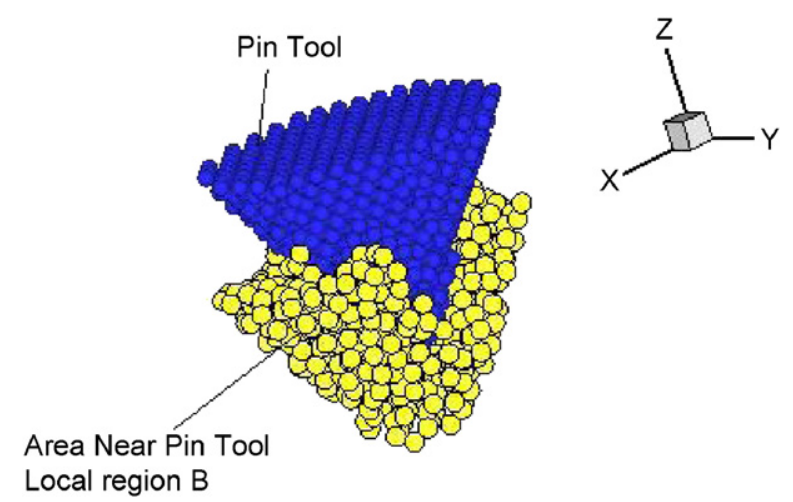

Fig. 5. Atom configuration of pin tool and local region B.

a

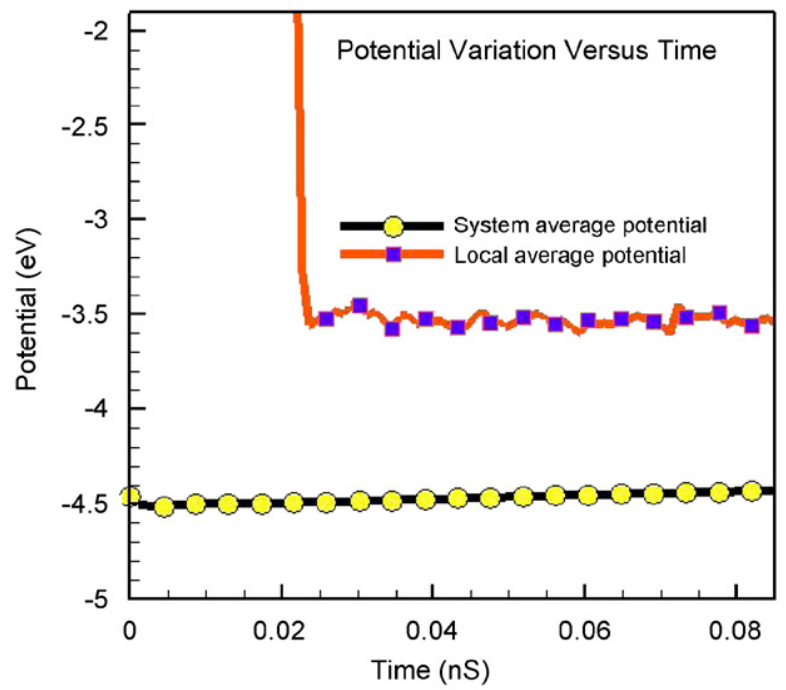

b

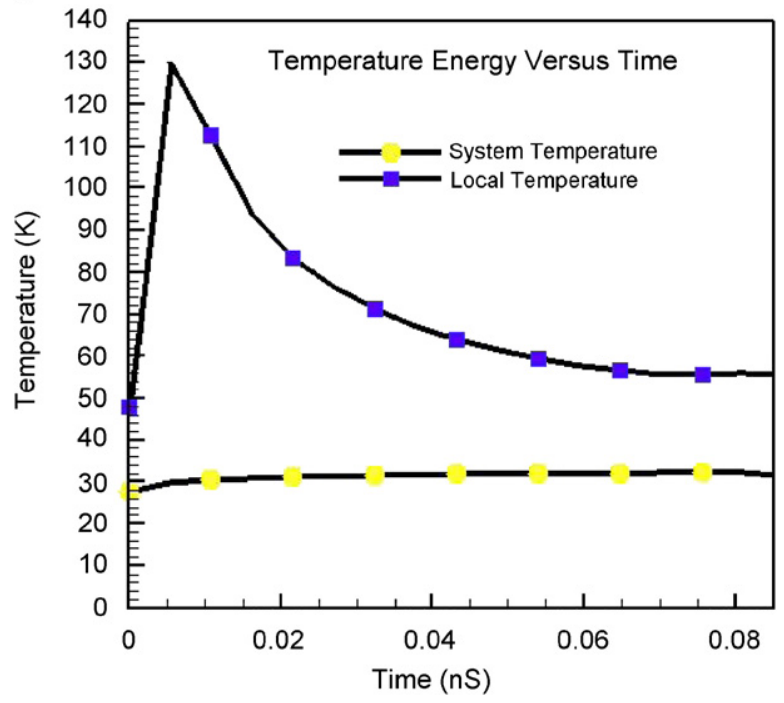

Fig. 6. (a) Potential energy evolution versus time. (b) Comparison of temperature at local zone B under pin tool with that of workpiece. 
obvious variation of potential energy in the local region $\mathrm{B}$. Before 0.02 ns (about 20000 MD steps), both workpiece and pin tool are in the states of separation as Fig. 1, the pin tool region is not formed and potential energy is zero. From 0.02 to $0.08 \mathrm{~ns}$, the potential energy is about $-3.6 \mathrm{eV}$, and the curve of potential energy is relatively smooth. It verifies that the cutting process is in the stable state. The potential energy in the tip region is greater than that of the system.

Fig. 6b shows the temperature comparison between inwhole system and local region B. Our simulation is under the NVT ensemble, the environment temperature is $30 \mathrm{~K}$. The temperature can be calculated from

$T=\left\langle\sum_{i=1}^{N} m_{i} v_{i}^{2}\right\rangle / 3 N \kappa_{\mathrm{B}}$

where $\kappa_{\mathrm{B}}$ is the Boltzmann constant, $N$ is atom number, $m_{i}$ and $v_{i}$ are the $i$ th atom mass and velocity, respectively, and 〈) denotes statistical averaging over all simulation time $[28,29]$. The system temperature is $30 \mathrm{~K}$ in the stable state, and the temperature in the pin tool region $\mathrm{B}$ is up to $130 \mathrm{~K}$ at $0.005 \mathrm{~ns}$, about five times higher than the environment temperature. After that time, the local temperature decreases gradually with time and is higher than the system temperature.

Temperature effect on dislocation motion is important, and some analysis was made. Hertzberg [30] pointed out that the Peierls resistance of dislocation motion is larger for covalence bond materials, and the yield strength is sensitive to temperature. The detailed measurements of the temperature dependence of the Peierls stress in silicon was given by Duesbery and Joos [27]. They pointed out that the Peierls stress at the DBTT of $820 \mathrm{~K}$ is $0.0073 \mu$ $(\mu=68.1 \mathrm{GPa})$, linear extrapolation of the results to $0 \mathrm{~K}$ gives a value of $0.15 \mu$. The similar analysis method can be applied to our MD simulation at the local temperature with the maximum $130 \mathrm{~K}$, the calculated stress is $0.0226 \mu=1.498 \mathrm{GPa}$. It means that as the shear stress is greater than $1.498 \mathrm{GPa}$ at $130 \mathrm{~K}$, the dislocation motion may occurred. However, based on our MD simulation, the shear stresses acting on dislocation is $0.4 \mathrm{GPa}$ at $t=$ $0.005 \mathrm{~ns}$ which is lower than $1.498 \mathrm{GPa}$. So it is reasonable that there is no dislocation motion observed in our present MD simulation.

Compared to our previous work without introducing a dislocation in the simulation model with the same temperature of $30 \mathrm{~K}$, a displace phase transformation of $\mathrm{Si}$ is observed, and there is no dislocation nucleation observed [28]. Both simulation results show that the pressure is big enough and the shear stress is relatively small.

\section{Conclusion}

MD simulation is carried out in order to understand the atomic scale mechanism of a pin tool cutting on a semiconductor silicon. The simulation shows that the cutting force is higher than both the thrust force and the width force.
From our work, one can learn that the pin tip scanned with a constant load does not make the dislocation motion in silicon, it is attributed to the small shear stress acting on the dislocations. And if the environment is under a lower temperature than that of the brittle-ductile transition, the evolution of transition from ductile to brittle is not easy to occur. It can provide valuable reference for designing nanometer devices which performance is sensitive to the motion and core position of dislocation, such as optoelectronic device [14]

\section{Acknowledgments}

The research presented here was supported by the National Natural Science Foundation of China (Grant nos. 10372107 and 10721202) and Chinese Academy of Sciences (Grant no. KJCX2-YW-M04), and Computer Network Information Center, Chinese Academy of Sciences (CAS).

\section{References}

[1] George A, Michot G. Dislocation loops at crack tips: nucleation and growth-an experimental study in silicon. Mater Sci Eng A 1993; $164: 118-34$

[2] Rabier J, Denanot MF, Demenet JL, Cordier P. Plastic deformation by shuffle dislocations in silicon. Mater Sci Eng A 2004;387-389:124-8.

[3] Saka H, Abe S. FIB/HVEM observation of the configuration of cracks and the defect structure near the crack in Si. J Electron Microsc 1997;46:45-57.

[4] Asaoka K, Umeda T, Arai S, Saka H. Direct evidence for shuffle dislocations in Si activated by indentations at 77K. Mater Sci Eng A 2005;400-401:93-6.

[5] Kaxiras E, Duesbery M. Free energy of generalized stacking faults in Si and implications for the brittle-ductile transition. Phys Rev Lett 1993;70:3752-5.

[6] Perez R, Gumbsch P. Direct anisotropy in the cleavage fracture os silicon. Phys Rev Lett 2000;84:5347-50.

[7] Bernstein N, Hess DW. Lattice trapping barriers to brittle fracture. Phys Rev Lett 2003;91:025501-4.

[8] Tanaka M, Higashida $K$, Nakashima $H$, Takagi $H$, Fujiwara $M$ Fracture toughness evaluated by indentation methods and its relation to surface energy in silicon single crystals. Mater Trans 2003;44:681-4.

[9] Tang CY, Zhang LC. A molecular dynamics analysis of the mechanical effect of water on the deformation of silicon monocrystals subjected to nano-indentation. Nanotechnology 2005;16:15-20.

[10] Garcia R, Perez R. Dynamic atomic force microscopy methods. Surf Sci Rep 2002;47:197-301.

[11] Lantz MA, Hug HJ, van Schendel PJA, Hoffmann R, Martin S, Baratoff $\mathrm{S}$, et al. Low temperature scanning force microscopy of the $\mathrm{Si}(111)$ $(7 \times 7)$ surface. Phys Rev Lett 2000;84:2642-5.

[12] Giessibl FJ, Hembacher S, Bielefeldt H, Mannhart J. Subatomic features on the silicon $(111)-(7 \times 7)$ surface observed by atomic force microscopy. Science 2000;289:422-5.

[13] Oral A, Grimble RA, Ozgur Ozer H, Hoffmann PM, Pethica JB. Quantitative atom-resolved force microscopy. Appl Phys Lett 2001;79:1915-7.

[14] Ren Q, Joos B, Duesbery MS. Test of the Peierls-Nabarro model for dislocation in silicon. Phys Rev B 1995;52:13223-8.

[15] Fang TH, Weng CI, Chang JG. Molecular dynamicsimulation of nanolithography process using atomic force microscopy. Surf Sci 2002;501:138-47.

[16] Haile JM. Molecular dynamics simulation: elementary methods New York: Wiley; 1992.

[17] Cheong WCD, Zhang LC. Molecular dynamics simulation of phase transformations in silicon monocrystals due to nano-indentation. Nanotechnology 2000;11:173-80.

[18] Isono Y, Tanaka T. Three-dimensional molecular dynamics simulation of atomic scale precision processing using a pin tool. JSME Int J Ser A 1997;40:211-8. 
[19] Tersoff J. Modelling solid-state chemistry: interatomic potentials for multicomponent system. Phys Rev B 1989;39:5566-8.

[20] Tersoff J. New empirical model for the structural properties of silicon. Phys Rev Lett 1986;36:632-5.

[21] Stillinger FH, Weber TA. Computer simulation of local order in condensed phases of silicon. Phys Rev B 1985;31:5262-71.

[22] Allen MP, Tildesley DJ. Computer simulation of liquid, vol. 340. New York:Oxford University Press; 2000.

[23] Kim YS, Lee YM, Choi DY, Kim CI. Quasimolecular dynamics simulation for bending fracture of laminar composite materials. J Mater Sci Technol 1980;29:41-6.

[24] Kim YS, Choi SO, Lee SR, Kim J. An atomic simulation of AFM-based nano lithography process for nano patterning. In: IUTAM symposium on mesoscopic dynamics of fracture process and materials strength, Japan, 6-11 July, 2003. p. 331-41.
[25] Hornstra J. Dislocation in the Diamond Lattice. J Phys Chem Solids 1958;5:129-41.

[26] Hirth JP, Lothe J. Theory of Dislocations. 2nd ed. New York: Wiley; 1982.

[27] Duesbery MS, Joos B. Dislocation motion in silicon: the shuffle-glide controversy. Philos Mag Lett 1996;74:253-8.

[28] Tang $Q$, Chen F. MD simulation of phase transformations due to nanoscale cutting on silicon monocrystals with diamond tip. J Phys D, Appl Phys 2006;39:3674-9.

[29] Wang X, Hu H, Xu X. Photo-acoustic measurement of thermal conductivity of thin films and bulk materials. J Heat Transfer 2001;123:138-44.

[30] Hertzberg RW. Deformation and fracture mechanics of engineering materials. New york: Wiley; 1976 [chapter 2]. 